\title{
Space-borne Bose-Einstein condensation for precision interferometry
}

\author{
Dennis Becker ${ }^{1,16}$, Maike D. Lachmann ${ }^{1,16}$, Stephan T. Seidel ${ }^{1,15,16}$, Holger Ahlers ${ }^{1}$, Aline N. Dinkelaker ${ }^{2}$, Jens Grosse ${ }^{3,4}$, \\ Ortwin Hellmig ${ }^{5}$, Hauke Müntinga ${ }^{3}$, Vladimir Schkolnik ${ }^{2}$, Thijs Wendrich ${ }^{1}$, André Wenzlawski $^{6}$, Benjamin Weps $^{7}$, \\ Robin Corgier ${ }^{1,8}$, Tobias Franz ${ }^{7}$, Naceur Gaaloul ${ }^{1}$, Waldemar Herr ${ }^{1}$, Daniel Lüdtke ${ }^{7}$, Manuel Popp ${ }^{1}$, Sirine Amri ${ }^{8}$, \\ Hannes Duncker ${ }^{5}$, Maik Erbe ${ }^{9}$, Anja Kohfeldt ${ }^{9}$, André Kubelka-Lange ${ }^{3}$, Claus Braxmaier ${ }^{3,4}$, Eric Charron ${ }^{8}$, Wolfgang Ertmer ${ }^{1}$, \\ Markus Krutzik ${ }^{2}$, Claus Lämmerzahl ${ }^{3}$, Achim Peters ${ }^{2}$, Wolfgang P. Schleich ${ }^{10,11,12,13}$, Klaus Sengstock ${ }^{5}$, Reinhold Walser ${ }^{14}$, \\ Andreas Wicht ${ }^{9}$, Patrick Windpassinger ${ }^{6} \&$ Ernst M. Rasel ${ }^{1 *}$
}

\begin{abstract}
Owing to the low-gravity conditions in space, space-borne laboratories enable experiments with extended free-fall times. Because Bose-Einstein condensates have an extremely low expansion energy, space-borne atom interferometers based on Bose-Einstein condensation have the potential to have much greater sensitivity to inertial forces than do similar ground-based interferometers. On 23 January 2017, as part of the sounding-rocket mission MAIUS1 , we created Bose-Einstein condensates in space and conducted 110 experiments central to matter-wave interferometry, including laser cooling and trapping of atoms in the presence of the large accelerations experienced during launch. Here we report on experiments conducted during the six minutes of in-space flight in which we studied the phase transition from a thermal ensemble to a Bose-Einstein condensate and the collective dynamics of the resulting condensate. Our results provide insights into conducting cold-atom experiments in space, such as precision interferometry, and pave the way to miniaturizing cold-atom and photon-based quantum information concepts for satellite-based implementation. In addition, space-borne Bose-Einstein condensation opens up the possibility of quantum gas experiments in low-gravity conditions ${ }^{1,2}$.

Studies of quantum systems such as matter-waves in the presence of a gravitational field ${ }^{3}$ can help to improve our understanding of general relativity ${ }^{4}$ and quantum mechanics. Because the sensitivity of measuring inertial forces with matter-wave interferometers is proportional to the square of the time that the atoms spend in the interferometer ${ }^{5}$, an extended free-fall of atoms in the interferometer results in a large enhancement in sensitivity ${ }^{1,6}$. In this context, slowly spreading ensembles with pico- or femtokelvin-scale expansion energies, obtained by Bose-Einstein condensation ${ }^{7,8}$ in combination with 'delta-kick' collimation $^{9-11}$, remain in the interferometer for longer and are therefore essential for interferometry over timescales of the order of tens of seconds. The associated large coherence lengths of the ensemble are needed to combine precision with accuracy ${ }^{1}$.
\end{abstract}

Generating and manipulating Bose-Einstein condensates (BECs) with low expansion energies is difficult because they can easily be compromised by experimental imperfections, very small forces and gravity. By creating a BEC on board a sounding rocket, we successfully demonstrated key atom-optics methods under microgravity conditions. Our experimental apparatus ${ }^{12-14}$ (Fig. 1) is equipped with a multilayer atom chip ${ }^{15-17}$. Its in-flight performance compares well with groundbased demonstrations, producing about $10^{5}$ atoms in $1.6 \mathrm{~s}$. This flux made it possible to perform a large number of experiments during the space flight, exemplified here by images of a space-based BEC (Fig. 1e) and of Bragg scattering of a BEC (Fig. 1f). The latter shows the spatial density profile of the BEC and its replica, which was generated by Bragg scattering at a light crystal and moves with a relative velocity that corresponds to the transfer of two photon recoils. In Fig. If we compare the size of the BEC in terms of the Thomas-Fermi radius and its separation from its replica $70 \mathrm{~ms}$ after the Bragg scattering event, which occurred $15.6 \mathrm{~ms}$ after the release of the BEC from the atom chip. The expansion velocity of the $\mathrm{BEC}$ is nine times smaller than the velocity that is transferred during Bragg scattering. The stripe pattern results from an intensity modulation of the light fields that induce the Bragg scattering.

In Fig. 2 we summarize the experiments of the MAIUS- 1 mission that were performed in space and during the launch of the rocket. These experiments build on those of the QUANTUS collaboration ${ }^{18,19}$, and complement those on dual-species interferometry ${ }^{20}$ and those that involve clocks based on laser-cooled atoms ${ }^{21}$. They are also instrumental for NASA's Cold Atom Laboratory ${ }^{2}$ (CAL) on the International Space Station (ISS) and for the NASA-DLR Bose-Einstein Condensate and Cold Atom Laboratory (BECCAL) multi-user facility, which is currently in the planning phase $\mathrm{e}^{22}$.

Here we report on BEC experiments with rubidium-87 atoms in space. We studied the phase transition from a thermal ensemble to a BEC by adjusting the temperature via forced radio-frequency evaporation of thermal atoms out of the atom-chip magnetic trap. In Fig. 3a we show the spatial atomic density of the thermal ensemble and the BEC at three different final radio frequencies of the forced evaporation (at the final cooling step). During the phase transition, with decreasing temperature the number of atoms in the thermal ensemble (extracted using a Gaussian fit, red curve in Fig. 3a) decreases markedly whereas that in the BEC increases (parabolic fit, blue curve in Fig. 3a). In Fig. 3b, c we compare the formation of BECs in space and on the ground; we also plot the fraction of atoms in the BEC with respect to the total atom number.

The comparison reveals that, for the same final radio frequency, the observed ratio of thermal and condensed atoms (and hence the fraction of the total number of atoms in the BEC) was lower in space than on the ground. We suspect that this difference is due to a change in the magnetic field in space with respect to that on the ground, resulting from, for example, a thermal drift in the current supply. In addition, the numbers of atoms in the thermal ensemble and in the BEC in space

\footnotetext{
${ }^{1}$ Institute of Quantum Optics, QUEST-Leibniz Research School, Leibniz University Hannover, Hanover, Germany. ${ }^{2}$ Department of Physics, Humboldt-Universität zu Berlin, Berlin, Germany. ${ }^{3}$ Center of Applied Space Technology and Microgravity (ZARM), University of Bremen, Bremen, Germany. ${ }^{4}$ Institute of Space Systems, German Aerospace Center (DLR), Bremen, Germany. ${ }^{5}$ nstitute of Laser-Physics, University Hamburg, Hamburg, Germany. ${ }^{6}$ Institute of Physics, Johannes Gutenberg University Mainz (JGU), Mainz, Germany. ${ }^{7}$ Simulation and Software Technology, German Aerospace Center (DLR), Brunswick, Germany. ${ }^{8}$ Institut des Sciences Moléculaires d'Orsay (ISMO), CNRS, Université Paris-Sud, Université Paris-Saclay, Orsay, France. ${ }^{9}$ Ferdinand-Braun-Institut, Leibniz-Institut für Höchstfrequenztechnik, Berlin, Germany. ${ }^{10}$ Institut für Quantenphysik and Center for Integrated Quantum Science and Technology (IQST), UIm, Germany. ${ }^{11}$ Hagler Institute for Advanced Study, Texas A\&M University, College Station, TX, USA. ${ }^{12}$ Texas A\&M AgriLife Research, Texas A\&M University, College Station, TX, USA. ${ }^{13}$ Institute for Quantum Science and Engineering (IQSE), Department of Physics and Astronomy, Texas A\&M University, College Station, TX, USA. ${ }^{14}$ Institut für Angewandte Physik, Technische Universität Darmstadt, Darmstadt, Germany. ${ }^{15}$ Present address: OHB System AG, Weßling, Germany. ${ }^{16}$ These authors contributed equally: Dennis Becker, Maike D. Lachmann, Stephan T. Seidel. *e-mail: rasel@iqo.uni-hannover.de
} 

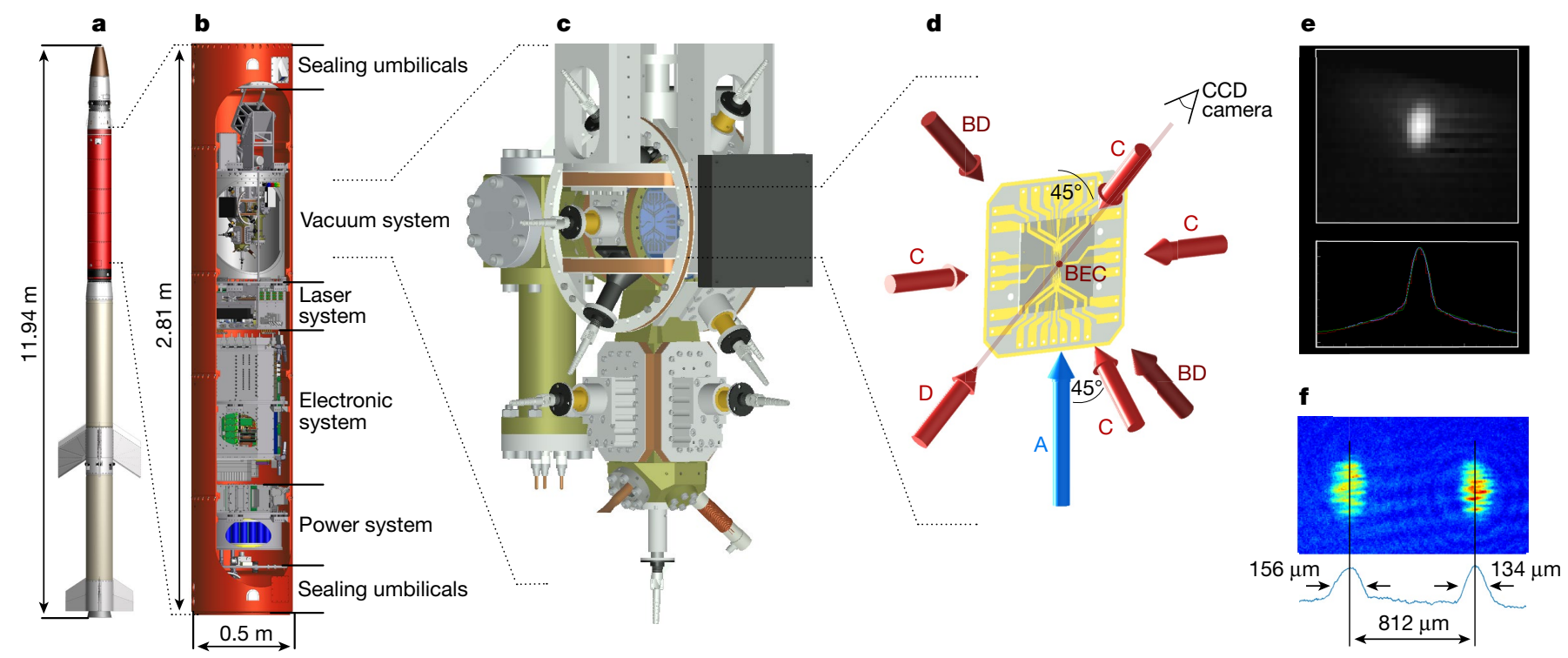

Fig. 1 | Set-up for space-borne Bose-Einstein condensation. a-d, The rocket (a) carried the payload (b), including the vacuum system (c) that houses the atom chip (d), into space. On the atom chip, a magneto-optical trap formed by laser beams $(\mathrm{C})$ is first loaded from the cold atomic beam (A). Afterwards, the BEC is created in, transported by and released from the magnetic trap of the atom chip. Two additional light beams (BD) induce Bragg diffraction, and a charge-coupled device (CCD) camera records the absorption image of the BEC using laser light (D). e, Grey-scale absorption image of the spatial density of the

are $64 \%$ higher than those obtained on the ground. This improvement in the BEC flux is most probably due to more efficient loading into the magnetic trap in the absence of gravitational sag. To optimize the BEC flux even further, the circuitry of the multilayer atom chip offers various trap configurations, with variable volume and depth. However,

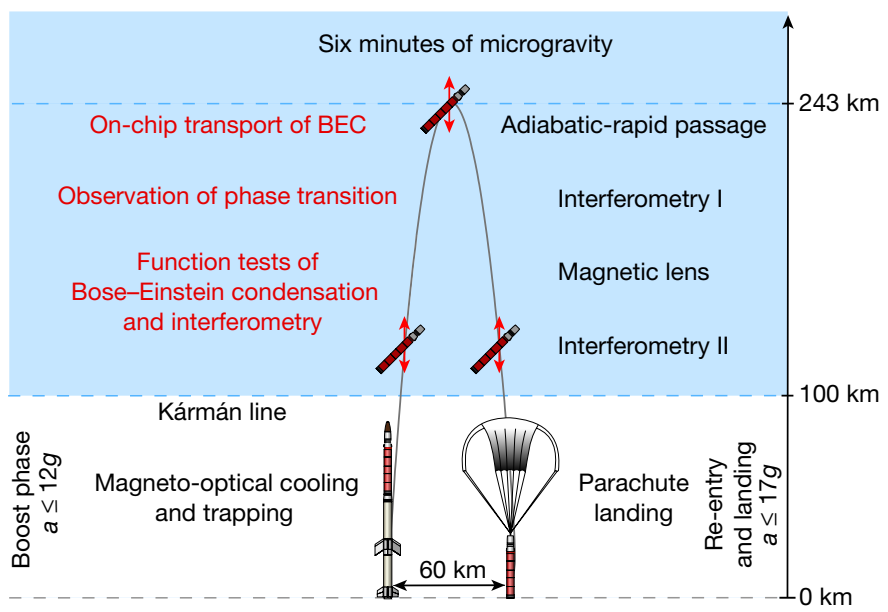

Fig. 2 Schedule for the MAIUS-1 sounding-rocket mission. During the boost phase (bottom left) and the $6 \mathrm{~min}$ of space flight (blue-shaded region), 110 atom-optics experiments were performed. Those discussed here are printed in red. In space (above the Kármán line, $100 \mathrm{~km}$ above the ground), inertial perturbations are reduced to a few parts per million of gravity, the pointing of the length axis is stabilized with respect to gravity (indicated by the red arrows) and the spin of the rocket is suppressed to about $5 \mathrm{mrad} \mathrm{s}^{-1}$ owing to rate control. During re-entry, the peak forces on the payload $(a)$ exceed the gravitational force on the ground $(g)$ by a factor of up to 17.
BEC in space (top; white corresponds to the highest densities) and its one-dimensional density profile (bottom; integrated from the top to the bottom of the image), which were sent to ground control in low resolution. f, Our demonstration of Bragg scattering, apparent in the momentum distribution of the BEC, opens up a path towards atom interferometry in space. The image contrasts the size of the BECs in the spatial superposition that we created with their relative separation $70 \mathrm{~ms}$ after the transfer of two photon recoils onto the replica, which moves to the right. The colour scale shows the spatial density of the clouds (blue, low; red, high).

experiments of this kind require more time than was available during our flight.

Because transporting and shaping BECs to create compact wavepackets are key to interferometry, we investigated the evolution of the BEC in free fall after release and the transport of BECs on the atom chip away from the surface of the chip via its impact on the BEC motion in free fall. In space, and therefore in the absence of gravitational sag, we can compare the predictions of a theoretical simulation directly with the observations. The BECs were moved across a distance of $0.8 \mathrm{~mm}$ from the surface of the chip. For this purpose, the homogeneous magnetic field, which in combination with the atom chip determines the location of the Ioffe-Pritchard trap, was lowered smoothly over $50 \mathrm{~ms}$ with a sigmoidal time dependence.

In particular, we studied oscillations in the centre-of-mass position of a BEC excited by its transport on the atom chip. For this purpose, the BEC was kept trapped for variable hold times of up to $25 \mathrm{~ms}$ before it was released. In Fig. $4 \mathrm{a}$ we show the positions with respect to the surface of the chip of BECs detected $50 \mathrm{~ms}$ after release as a function of hold time; these positions reflect the varying initial velocities of the BECs due to their centre-of-mass oscillation. Using these data, we can reconstruct the motion of the BEC in the trap. Of a total of ten measurements, five tested the repeatability of the preparation for zero hold time (Fig. 4a, green circles) and five probed the oscillatory behaviour (black circles) for increasing hold time; the latter illustrate the sinusoidal dependence of the distance of the centre-of-mass of the BEC from the chip on hold time, over various trials, consistent with the fitted sinusoidal behaviour (dashed purple line) of a trapped quantum gas.

In addition, we investigated the motion of the BEC for times of up to $300 \mathrm{~ms}$ after release after zero hold time. We include the data from Fig. 4a for $50 \mathrm{~ms}$ after release (green circles) also in Fig. 4b. According to Fig. $4 \mathrm{a}$, the velocity of the BEC associated with the oscillation is maximum for zero hold time. This velocity adds to that due to transport away from the chip; hence, the total velocity of the BEC after release 

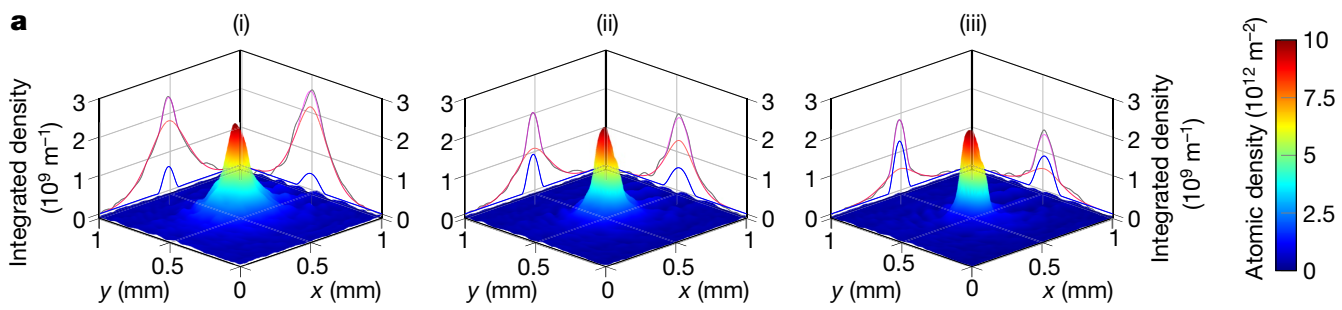

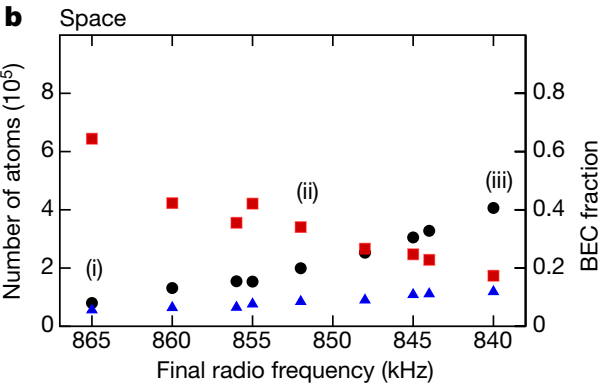

- Thermal ensemble

Fig. 3 Phase transition to the BEC in space and on the ground, controlled by the final radio frequency of the forced evaporation. a, Spatial atomic density (colour scale) and corresponding line integrals (solid grey lines), as well as Gaussian (red lines) and parabolic (blue lines) fits of the line integrals of the thermal and condensed atoms, respectively, and their sum (violet lines), for cases in space where 8\% (i), $20 \%$ (ii) and $41 \%$ (iii) of the atoms are in the BEC state. b, c, The number of magnetically trapped atoms in the thermal ensemble (red squares,

was as large as $8.8 \mathrm{~mm} \mathrm{~s}^{-1}$, as inferred from a linear fit (dashed purple line in Fig. 4b). Moreover, after release, we transferred the atoms by adiabatic rapid passage into a mixture of the Zeeman states so that we could detect possible residual fields. Despite the strong effect of preparation and transport on the motion of the BEC, the trajectories demonstrate only a small scatter in the experimental data for different Zeeman states of the $F=2$ manifold (Fig. $4 \mathrm{~b}$; triangles, $m_{F}=0$; circles, $m_{F}=2 ; m_{F}$ is the magnetic angular momentum quantum number; $F$ is the hyperfine splitting quantum number) and set an upper bound of $1 \%$ for the corresponding relative fluctuation in the initial velocity that is possibly caused by the various manipulations of the atom chip. In addition to minimizing the amplitude of the centre-of-mass oscillations, phase stability of these oscillations is required for quantum tests such as those proposed for the STE-QUEST satellite mission ${ }^{1}$.

We compare our measurements to a theoretical model ${ }^{23}$ of the dynamics of the BEC after creation, including its oscillation in the trap, release and evolution until detection. This model includes the current-carrying wire structures of the experimental set-up and solves the Gross-Pitaevskii equation in the Thomas-Fermi regime ${ }^{24}$. Our experimental results shown in Fig. $4 \mathrm{a}$, b agree well with our simulation (solid blue lines), differing only by a slight underestimate of the oscillation amplitude and a corresponding small velocity offset. This difference may result from the model for shutting off the release trap as we have limited knowledge of the magnetic field dynamics during the switchoff. Most other potential reasons are excluded by the simulation, which allows us to check for the influence on the BEC dynamics of the circuitry of the atom chip, of the Helmholtz coils and of uncertainties in the current values (shaded areas in Fig. 4).

The motion on the atom chip causes complicated oscillations in the shape of the $\mathrm{BEC}^{25}$ (Fig. 4c); this finding again demonstrates the importance of phase-stable manipulations. Our theoretical simulations show that the observed variations in the size of the BEC as defined by the Thomas-Fermi radii originate from oscillations in the shape of the BEC rather than from experimental noise, as confirmed by the low phase scatter at release (Fig. 4d), with relative fluctuations of only about $2 \%$. Although the density of the BEC reduces by one order of magnitude during transport, the remaining mean-field energy still causes the BEC

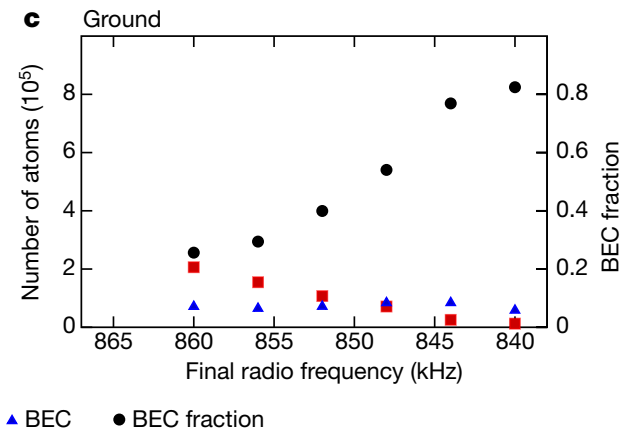

left axis) is higher in space (b) than on the ground (c), resulting in more atoms in the BEC (blue triangles, left axis) in space; for a comparable BEC fraction, there are $64 \%$ more atoms in the BEC in space than in the BEC on the ground. The dependence of the fraction of the total number of atoms in the BEC (that is, the number of atoms in the BEC divided by the sum of the numbers of atoms in the BEC and in the thermal ensemble; black circles, right axis) on the radio frequency is also different in space and on the ground. In $\mathbf{b}$, cases (i)-(iii) from $\mathbf{a}$ are indicated for reference.

to expand by up to about $1 \mathrm{~mm}$ in diameter after $300 \mathrm{~ms}$ (Fig. 4d), in agreement with our theoretical prediction (solid blue and red lines). The thermal background of the released atoms has a temperature of approximately $100 \mathrm{nK}$ and the residual expansion of the BEC corresponds to a kinetic energy equivalent to a few nanokelvin, as expected from the decompression during transport. Further reduction of the expansion energies requires additional measures, such as delta-kick collimation, which could not be studied during the short rocket flight.

The phase stability of the centre-of-mass motion and of the collective size oscillations of the BEC is necessary to reproducibly release the atoms into free fall with vanishing velocity and to lower the expansion to the required level. To minimize the release velocity, we propose transport protocols that largely suppress the amplitude of the sinusoidal oscillations, bringing into reach BECs with release velocities below micrometres per second. Low expansion rates in all three dimensions, corresponding to kinetic energies of a few tens of picokelvin, are possible with atom chips by combining delta-kick collimation with excitation of a quadrupole shape oscillation ${ }^{23}$. The latter compensates for the asymmetric trap of the atom chip favouring the implementation of cylindrical lenses.

In conclusion, we used a payload on board a sounding rocket to create BECs in space. Although many experiments were performed during this space flight, here we focus on studies of the phase transition of the thermal ensemble to a BEC and the collective oscillations of the centre-of-mass and size of the BEC in the trap induced by the transport of the BEC away from the atom chip. The reproducibility that we have demonstrated allows the implementation of more sophisticated transport protocols (such as shortcut-to-adiabaticity protocols ${ }^{23}$ or optimal control protocols), and enables shape oscillations to be used jointly with delta-kick collimation to reduce and shape the expansion of BECs, to extend the time that they spend in the interferometer. Our experiments demonstrate the atom-optics tools that are required for satellite gravimetry ${ }^{26}$, for quantum tests of the equivalence principle ${ }^{27}$ and for gravitational-wave detection based on matter-wave interferometry in space $^{28}$. Moreover, they pave the way to miniaturizing cold-atom and photon-based quantum information concepts, and to integrating these concepts into quantum-communication satellites ${ }^{29-33}$. 

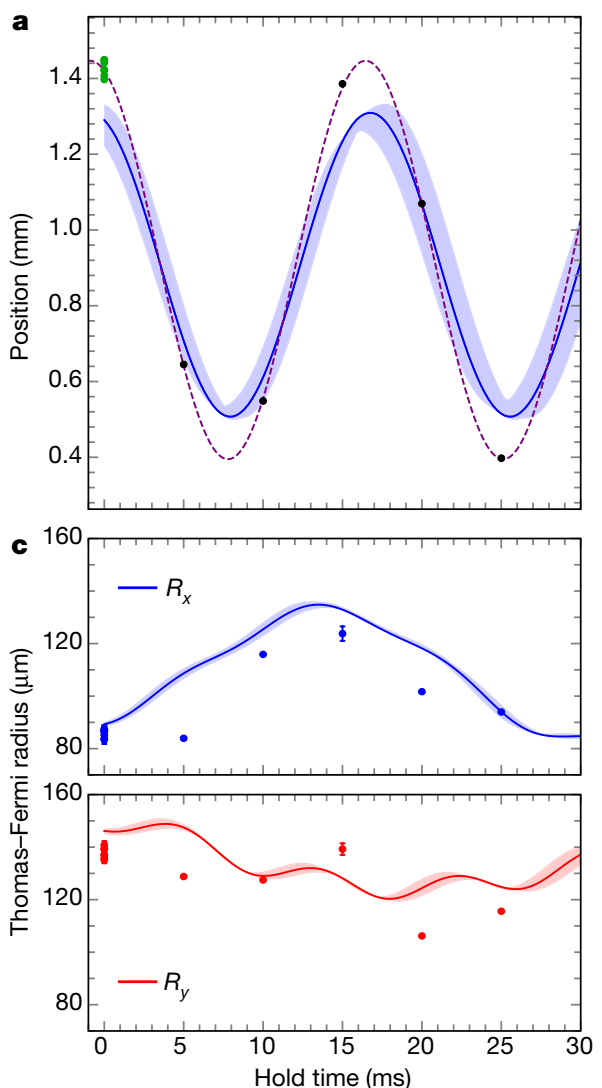

Fig. 4 Excitation of the centre-of-mass motion and oscillations in the shape of a space-borne BEC as a result of its transport away from an atom chip. a, From the modulation of the distance travelled by the BEC $50 \mathrm{~ms}$ after its release for different hold times, we infer the centre-ofmass motion of the BEC in the trap as a function of hold time by fitting a sinusoid (purple dashed line) to the data (green and black circles for immediate release and varying hold times, respectively). The simulation of the evolution of the BEC (blue line) agrees well with the data, but underestimates the amplitude of the oscillation. $\mathbf{b}$, The centre-of-mass motion of the BEC away from the atom chip after release from the trap is well fitted by a linear function (purple dashed line; purple shading indicates the $95 \%$ confidence interval), and is almost identical for different Zeeman states of the $F=2$ manifold (grey triangles, $m_{F}=0$; black and green circles, $m_{F}=2$; green circles in $\mathbf{a}$ and $\mathbf{b}$ represent the same data). The simulation of the dynamics of the BEC based on the Gross-Pitaevskii equation in the Thomas-Fermi limit is also shown (blue line). The inset shows a close-up of the boxed region of the main plot. $\mathbf{c}$, The Thomas-

\section{Online content}

Any methods, additional references, Nature Research reporting summaries, source data, statements of data availability and associated accession codes are available at https://doi.org/10.1038/s41586-018-0605-1.

Received: 18 June 2018; Accepted: 9 August 2018; Published online 17 October 2018.

1. Aguilera, D. N. et al. STE-QUEST_test of the universality of free fall using cold atom interferometry. Class. Quantum Gravity 31, 115010 (2014).

2. Elliott, E. R., Krutzik, M. C., Williams, J. R., Thompson, R. J. \& Aveline, D. C. NASA's Cold Atom Lab (CAL): system development and ground test status. npj Microgravity 4, 16 (2018).

3. Colella, R., Overhauser, A. W. \& Werner, S. A. Observation of gravitationally induced quantum interference. Phys. Rev. Lett. 34, 1472-1474 (1975).

4. Misner, C. W., Thorne, K. S. \& Wheeler, J. A. Gravitation (Princeton Univ. Press, Princeton, 1973).

5. Berman, P. R. Atom Interferometry Ch. 9 (Academic Press, New York, 1997)

6. Dimopoulos, S., Graham, P. W., Hogan, J. M. \& Kasevich, M. A. Testing general relativity with atom interferometry. Phys. Rev. Lett. 98, 111102 (2007).

7. Cornell, E. A. \& Wieman, C. E. Nobel lecture: Bose-Einstein condensation in a dilute gas, the first 70 years and some recent experiments. Rev. Mod. Phys. 74, 875-893 (2002).
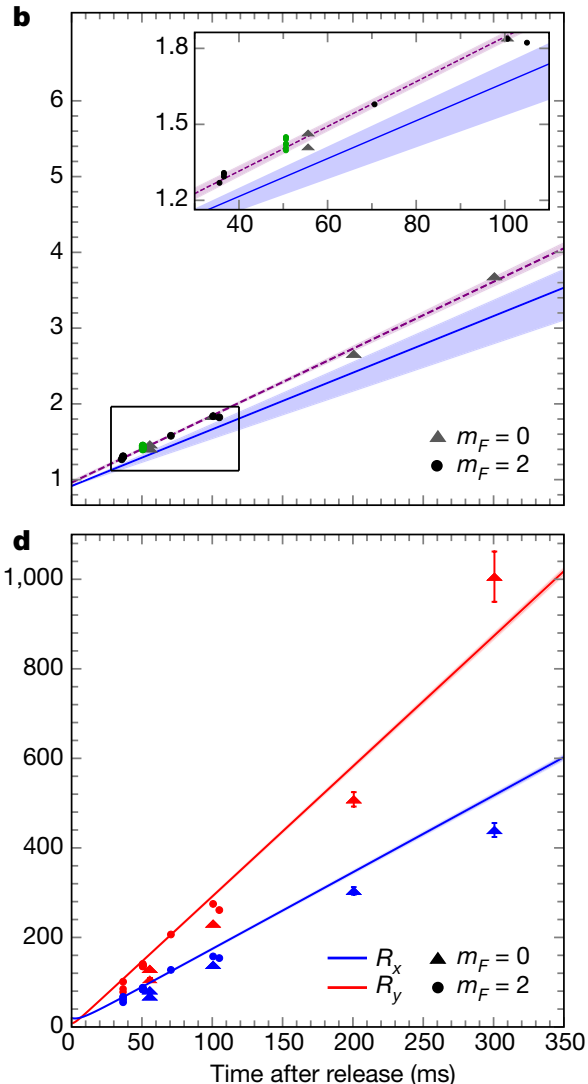

Fermi radii $R_{x}$ (top, blue circles) and $R_{y}$ (bottom, red circles) serve as measures of the size and thus the shape of the BEC $50 \mathrm{~ms}$ after release. For varying hold time, these radii display complicated oscillations, which also appear in our simulations (red and blue lines) of the BEC evolution. d, Thomas-Fermi radii for condensates that were released immediately after transport and freely expanded. After $300 \mathrm{~ms}$, the BEC has grown in size, up to about $1 \mathrm{~mm}$. Most experiments were performed with BECs in the $m_{F}=0$ state (blue and red triangles), with the results in accordance with our theory for BECs in the $m_{F}=0$ state (red and blue lines), but some were performed with BECs in the $m_{F}=2$ state (blue and red circles). Possible deviations due to residual magnetic field gradients are below the measurement resolution. In all panels, error bars indicate uncertainties related to fitting the images of the BECs. Uncertainties in the theoretical model (blue and red shaded areas) reflect the degree of knowledge of the experimental parameters, such as those related to the generation of a magnetic field by electrical circuits and currents (in particular, the range of currents used in the simulations; see Methods).

8. Ketterle, W. Nobel lecture: When atoms behave as waves: Bose-Einstein condensation and the atom laser. Rev. Mod. Phys. 74, 1131-1151 (2002).

9. Chu, S., Bjorkholm, J. E., Ashkin, A., Gordon, J. P. \& Hollberg, L. W. Proposal for optically cooling atoms to temperatures of the order of $10^{-6} \mathrm{~K}$. Opt. Lett. 11, 73-75 (1986).

10. Müntinga, H. et al. Interferometry with Bose-Einstein condensates in microgravity. Phys. Rev. Lett. 110, 093602 (2013).

11. Kovachy, T. et al. Matter wave lensing to picokelvin temperatures. Phys. Rev. Lett. 114, 143004 (2015).

12. Grosse, J. et al. Design and qualification of an UHV system for operation on sounding rockets. J. Vac. Sci. Technol. A 34, 031606 (2016).

13. Schkolnik, V. et al. A compact and robust diode laser system for atom interferometry on a sounding rocket. Appl. Phys. B 122, 217 (2016).

14. Kubelka-Lange, A. et al. A three-layer magnetic shielding for the MAIUS-1 mission on a sounding rocket. Rev. Sci. Instrum. 87, 063101 (2016).

15. Hänsel, W., Hommelhoff, P., Hänsch, T. W. \& Reichel, J. Bose-Einstein condensation on a microelectronic chip. Nature 413, 498-501 (2001).

16. Folman, R., Krüger, P., Schmiedmayer, J., Denschlag, J. \& Henkel, C. Microscopic atom optics: from wires to an atom chip. Adv. At. Mol. Opt. Phys. 48, 263-356 (2002).

17. Fortágh, J. \& Zimmermann, C. Magnetic microtraps for ultracold atoms. Rev. Mod. Phys. 79, 235-289 (2007).

18. van Zoest, T. et al. Bose-Einstein condensation in microgravity. Science $\mathbf{3 2 8}$, 1540-1543 (2010).

19. Rudolph, J. et al. A high-flux BEC source for mobile atom interferometers. New J. Phys. 17, 065001 (2015). 
20. Barrett, B. et al. Dual matter-wave inertial sensors in weightlessness. Nat. Commun. 7, 13786 (2016).

21. Liu, L. et al. In-orbit operation of an atomic clock based on laser-cooled ${ }^{87} \mathrm{Rb}$ atoms. Nat. Commun. 9, 2760 (2018).

22. Gibney, E. Universe's coolest lab set to open up quantum world. Nature 557, 151-152 (2018).

23. Corgier, R. et al. Fast manipulation of Bose-Einstein condensates with an atom chip. New J. Phys. 20, 055002 (2018).

24. Pethick, C. \& Smith, H. Bose-Einstein Condensation in Dilute Gases Ch. 6 (Cambridge Univ. Press, Cambridge, 2002)

25. Stringari, S. Collective excitations of a trapped Bose-condensed gas. Phys. Rev. Lett. 77, 2360-2363 (1996).

26. Douch, K., Wu, H., Schubert, C., Müller, J. \& dos Santos, F. P. Simulation-based evaluation of a cold atom interferometry gradiometer concept for gravity field recovery. Adv. Space Res. 61, 1307-1323 (2018).

27. Altschul, B. et al. Quantum tests of the Einstein equivalence principle with the STE-QUEST space mission. Adv. Space Res. 55, 501-524 (2015).

28. Hogan, J. M. et al. An atomic gravitational wave interferometric sensor in low earth orbit (AGIS-LEO). Gen. Relativ. Gravit. 43, 1953-2009 (2011).

29. Armengol, J. M. P. et al. Quantum communications at ESA: towards a space experiment on the ISS. Acta Astronaut. 63, 165-178 (2008)

30. Ren, J.-G. et al. Ground-to-satellite quantum teleportation. Nature 549, 70-73 (2017).

31. Liao, S.-K. et al. Satellite-to-ground quantum key distribution. Nature 549, 43-47 (2017)

32. Yin, J. et al. Satellite-based entanglement distribution over 1200 kilometers. Science 356, 1140-1144 (2017)

33. Yin, J. et al. Satellite-to-ground entanglement-based quantum key distribution. Phys. Rev. Lett. 119, 200501 (2017).

Acknowledgements This work is supported by the DLR Space Administration with funds provided by the Federal Ministry for Economic Affairs and Energy (BMWi) under grant numbers DLR 50WM1131-1137, 50WM0940 and 50WM1240. W.P.S. thanks Texas A\&M University for a Faculty Fellowship at the Hagler Institute for Advanced Study at Texas A\&M University and Texas A\&M AgriLife for support for this work. The research of the IQST is financed partially by the Ministry of Science, Research and Arts Baden-Württemberg.
N.G. acknowledges funding from Niedersächsisches Vorab through the Quantum- and Nano-Metrology (QUANOMET) initiative within the project QT3. W.H. acknowledges funding from Niedersächsisches Vorab through the project Foundations of Physics and Metrology project. R.C. is a recipient of DAAD (Procope action and mobility scholarship) and a member of the IP@ Leibniz programme, which is supported by LU Hanover. S.T.S. is grateful for non-monetary support from DLR MORABA before, during and after the MAIUS-1 launch. We thank E. Kajari and M. Eckardt for the chip model code and A. Roura and W. Zeller for their input. We thank C. Spindeldreier and H. Blume from IMS Hanover for FPGA software development. We acknowledge the contributions of PTB Brunswick and LNOE Hanover towards fabricating the atom chip. We thank ESRANGE Kiruna and DLR MORABA Oberpfaffenhofen for assistance during the test and launch campaign.

Reviewer information Nature thanks L. Liu and the other anonymous reviewer(s) for their contribution to the peer review of this work.

Author contributions D.B., M.D.L., S.T.S., H.A., A.N.D., J.G., O.H., H.M., V.S., T.W., A.We., B.W., T.F., D.L., M.P., M.E., A.K., H.D., A.K.-L. and M.K. designed, built and tested the apparatus. D.B., M.D.L., H.A., A.N.D., J.G., O.H., H.M., V.S., T.W., A.We. and B.W., with S.T.S. as scientific lead, planned and executed the campaign. D.B., M.D.L. and S.T.S. evaluated the data. N.G., R.C., E.C., S.A., W.H. and D.B. carried out the simulations. E.M.R., W.P.S., M.D.L., D.B. and N.G. wrote the manuscript, with contributions from all authors. C.B., W.E., C.L., A.P., W.P.S., K.S., R.W., A.Wi. and P.W. are the co-principal investigators of the project, and E.M.R. its principal investigator.

Competing interests The authors declare no competing interests.

Additional information

Supplementary information is available for this paper at https://doi.org/ 10.1038/s41586-018-0605-1.

Reprints and permissions information is available at http://www.nature.com/ reprints.

Correspondence and requests for materials should be addressed to E.M.R Publisher's note: Springer Nature remains neutral with regard to jurisdictional claims in published maps and institutional affiliations. 


\section{METHODS}

Detection and analysis of absorption images. BECs were detected using an absorption-detection system that uses the $F=2 \rightarrow F^{\prime}=3$ transition of rubidium-87 atoms. A collimated detection beam enters the vacuum chamber via a window from one side, excites the atoms, and leaves the chamber at the opposite side. With two lenses and one mirror, the beam is directed onto the sensor of a CCD camera (pco.1400). During each sequence, one image including the shadow of the atoms and a later one without the shadow is recorded. Both are corrected with a dark image in the absence of the detection beam. The atomic column density is calculated from the optical density in MATLAB according to ref. ${ }^{34}$. Using a bimodal fit, consisting of a Thomas-Fermi and a Gaussian distribution, the number of atoms in the ensemble and its size and position are calculated.

Simulation. The theory curves in Fig. 4 are generated using a Gross-Pitaevskii equation solver, in the Thomas-Fermi regime, as described elsewhere ${ }^{3}$. The trapping frequencies are extracted from a detailed atom-chip simulation package, as used previously ${ }^{19}$. The chip structure is comparable to that obtained in ref. ${ }^{19}$ and involves two $z$ currents on two layers of the chip (base chip and science chip) and two currents through coils. Possible lack of knowledge of these currents is the origin of the shaded areas in Fig. 4. We scan these currents (from larger to smaller values) over the following intervals: $x$ coil, $[-0.02,-0.01] \mathrm{A} ; y$ coil, [-1.605, -1.595] A; base chip, [4.95, 5] A; science chip, [1.98, 2] A.

\section{Data availability}

Source Data for Figs. 3b, c and 4 are available with the online version of the paper.

34. Reinaudi, G., Lahaye, T., Wang, Z. \& Guéry-Odelin, D. Strong saturation absorption imaging of dense clouds of ultracold atoms. Opt. Lett. 32, 3143-3145 (2007). 